\title{
Design of a non-interventional post- marketing study to assess the long-term safety and effectiveness of ocrelizumab in German real world multiple sclerosis cohorts - the CONFIDENCE study protocol
}

Petra Dirks ${ }^{1}$, Vera Zingler ${ }^{2}$, Jost Leemhuis ${ }^{1}$, Heike Berthold ${ }^{1}$, Stefanie Hieke-Schulz ${ }^{1}$, David Wormser ${ }^{2}$ and Tjalf Ziemssen ${ }^{3^{*}}$

\begin{abstract}
Background: Multiple sclerosis (MS) is a chronic disease that requires lifelong treatment. A highly effective drug not only for relapsing but also for progressive forms of MS with a favorable safety profile is needed to further improve overall patient outcomes. Ocrelizumab, a recombinant humanized monoclonal antibody that selectively targets CD20-expressing B-cells, is the first drug indicated for the treatment of adult patients with relapsing forms of MS (RMS) and primary progressive MS (PPMS). Its safety and effectiveness profile has yet to be studied in a large, real-world setting. CONFIDENCE aims to further characterize the safety profile of ocrelizumab in routine clinical practice. In addition, real-world effectiveness data will be collected to complement the efficacy data documented in the pivotal clinical trials.

Methods: CONFIDENCE is a non-interventional, prospective, multicenter, long-term study collecting primary data from 3000 RMS and PPMS patients newly treated with ocrelizumab and 1500 patients newly treated with other selected MS disease-modifying therapies (DMTs). Treatment must be in accordance with the local label and follow routine practice. Data will be collected at approximately 250 neurological centers and practices across Germany. The recruitment period of 30 months started in April 2018. The observation period per patient is planned 7.5 to 10 years, depending on the date of inclusion, regardless of whether patients discontinue treatment. Visits follow routine practice and will be documented approximately every 6 months. The primary endpoint is the incidence and type of uncommon adverse events and death. Statistical analyses will be mainly descriptive and exploratory.

Discussion: CONFIDENCE is a large, non-interventional, post-authorization safety study that assesses long-term safety and effectiveness of ocrelizumab and other DMTs in a real-world setting. Data collected in CONFIDENCE will also be integrated into studies that have been developed to fulfil international regulatory requirements.
\end{abstract}

Keywords: Ocrelizumab, Relapsing multiple sclerosis, Primary progressive multiple sclerosis, Real world data, Noninterventional study, Long-term safety and effectiveness, Disease modifying therapy, MSDS3

\footnotetext{
* Correspondence: tjalf.ziemssen@uniklinikum-dresden.de

${ }^{3}$ Universitätsklinikum Carl Gustav Carus, Zentrum für klinische

Neurowissenschaften, Fetscherstr. 74, 01307 Dresden, Germany

Full list of author information is available at the end of the article
}

(C) The Author(s). 2020 Open Access This article is licensed under a Creative Commons Attribution 4.0 International License, which permits use, sharing, adaptation, distribution and reproduction in any medium or format, as long as you give appropriate credit to the original author(s) and the source, provide a link to the Creative Commons licence, and indicate if changes were made. The images or other third party material in this article are included in the article's Creative Commons licence, unless indicated otherwise in a credit line to the material. If material is not included in the article's Creative Commons licence and your intended use is not permitted by statutory regulation or exceeds the permitted use, you will need to obtain permission directly from the copyright holder. To view a copy of this licence, visit http://creativecommons.org/licenses/by/4.0/ The Creative Commons Public Domain Dedication waiver (http://creativecommons.org/publicdomain/zero/1.0/) applies to the data made available in this article, unless otherwise stated in a credit line to the data. 


\section{Background}

Multiple sclerosis (MS) is a chronic, inflammatory, demyelinating disease of the central nervous system (CNS) [1]. It presents as relapsing (RMS) or primary progressive MS (PPMS). Disease-modifying therapies (DMTs) approved for RMS reduce the number of relapses and slow progression of disease, thus delaying disability in RMS. Ocrelizumab (Ocrevus ${ }^{\circ}$ ) is the only drug approved for PPMS. DMTs modulate the immune system by various mechanisms of action, aiming to reduce the risk of inflammatory disease activity by suppressing peripheral lymphocyte activity affecting or infiltrating the CNS [2]. A concern of medication that targets the immune system is an increase in risk of serious infections and malignancy, especially with prolonged use [3]. MS requires a lifelong therapy; however, long-term use of MS therapies has been associated with the occurrence of serious side effects [4]. Thus, a highly effective drug with a favorable safety profile is still needed to further improve overall patient outcomes.

Ocrelizumab is a recombinant humanized monoclonal immunoglobulin G1 (IgG1) antibody designed to selectively target CD20-positive B cells [5]. In clinical trials, ocrelizumab demonstrated a favorable benefit/risk profile in RMS and PPMS patients [6, 7]. It was approved by the United States (U.S.) Food and Drug Administration (FDA) on 28 March 2017 as first medication for the treatment of adult patients with RMS as well as PPMS, and by the European Medicines Agency (EMA) on 08 January 2018 for treatment of active RMS and early PPMS.

Ocrelizumab demonstrated superior efficacy in a double blind, randomized phase II trial compared with placebo in relapsing-remitting MS (RRMS) (NCT00676715 [8];). Two identical 96-week, randomized, active-controlled phase III trials in RMS patients, OPERA I (NCT01247324) and OPERA II (NCT01412333), demonstrated that ocrelizumab is more efficacious than interferon (IFN) $\beta$-1a for the treatment of RMS [6]. A third pivotal double-blind, randomized, placebo-controlled phase III trial in PPMS patients, ORATORIO (NCT01194570), showed that ocrelizumab is more efficacious than placebo for the treatment of PPMS [7]. Results of these trials show that depletion of CD20-positive B cells leads to a significant impact on all measurable parameters of clinical and subclinical disease activity, including relapses, disability progression, and magnetic resonance imaging (MRI) outcomes related to disease progression.

Overall, ocrelizumab showed a favorable safety profile in RMS and PPMS patients. In phase III trials, proportions of adverse events (AEs) and serious AEs were comparable for ocrelizumab and comparator arms $[6,7]$. In RMS trials, fewer serious infections were reported for ocrelizumab-treated patients than IFN $\beta$-1a-treated patients (1.3\% vs. $2.9 \%)$; in PPMS trials, serious infections were similar between groups (6.2\% [ocrelizumab] and 5.9\% [placebo]). Pooled data from phase II trial NCT00676715, OPERA I and II, and ORATORIO implied an imbalance in malignancies in the ocrelizumab treatment group versus pooled IFN $\beta$-1a and placebo. The rate of malignancies in ocrelizumab-treated patients, however, remained within the range reported in epidemiological data $[6,7]$. Safety data from controlled treatment and open-label extension periods of the phase II and phase III trials are generally consistent with phase IIIb studies CHORDS (NCT0263785), VELOCE (NCT02545868), CASTING (NCT0286101), and OBOE (NCT0268898), which comprise more than 10,000 combined patient years of ocrelizumab exposure [9]. While the pivotal and phase IIIb studies established the safety and efficacy of ocrelizumab under controlled trial conditions in a selected patient population, data are needed to further characterize the safety and effectiveness of ocrelizumab over a long treatment duration and, importantly, in a clinical practice setting.

The non-interventional, post-authorization safety study (PASS) CONFIDENCE will capture long-term safety and effectiveness data of MS patients newly treated with ocrelizumab, i.e. exposed to ocrelizumab for the first time in their treatment history, in a real-world setting. To give this data context, CONFIDENCE will also collect data of MS patients newly treated with selected MS DMTs other than ocrelizumab in parallel. With regard to safety, the study aims to observe general long-term safety with a special focus on uncommon AEs (i.e. AEs with an incidence of 0.1 to $1 \%$ [ 1 to 10 out of 1000 patients] or less; e.g. serious infections and malignancies). In addition, ocrelizumab realworld effectiveness data will be collected over a long duration to complement the efficacy from phase III studies.

Collected safety data will be integrated into global ocrelizumab post-marketing safety studies, which have been developed to fulfil regulatory requirements of various health authorities. CONFIDENCE is thus the central study in the global ocrelizumab post-marketing safety program.

\section{Methods/design \\ Objectives}

The primary objective of CONFIDENCE is:

- To assess the long-term safety of ocrelizumab with special focus on the occurrence and characterization of uncommon AEs in patients with MS newly exposed to ocrelizumab.

The key secondary objective is:

- To assess the long-term safety of selected DMTs other than ocrelizumab with special focus on the occurrence and characterization of uncommon AEs in patients with MS exposed to those DMTs for the first time during the course of their MS therapy. 
Additional secondary objectives are:

- To estimate long-term effectiveness of ocrelizumab and of other selected DMTs, (no clinical disease activity measured by relapse and disease progression and no treatment discontinuation due to AEs [excluding pregnancies] and lack of effectiveness) in patients with MS newly exposed to ocrelizumab or to other selected DMTs.

- To estimate the incidence of serious infections, malignancies and the malignancy-related mortality rate in patients with MS newly exposed to ocrelizumab or to other selected DMTs.

\section{Study design}

CONFIDENCE is a long-term, prospective, multicenter, non-interventional study that collects primary safety and effectiveness data of patients with RMS and PPMS newly treated with ocrelizumab (ocrelizumab cohort) or patients newly treated with other selected MS DMTs (selected DMT cohort). Data from patients treated with other selected DMTs will be used to present ocrelizumab data in context of real-world MS treatment. These DMTs are alemtuzumab, cladribine, dimethyl fumarate, fingolimod, natalizumab and teriflunomide. In order to avoid the overrepresentation of individual DMTs other than ocrelizumab, an upper limit of 500 patients is defined for each them (see Study Population). The decision to treat with ocrelizumab or other DMT must be made by the treating physician before and independently of the decision to enroll the patient in the study. Dosing and treatment duration with ocrelizumab or other DMT will be at the discretion of the physician in accordance with local clinical practice as described in the summary of product characteristics $(\mathrm{SmPC})$ of ocrelizumab (Ocrevus $\left.{ }^{\circ}\right)$, alemtuzumab (Lemtrada ${ }^{\circ}$, cladribine (Mavenclad $\left.{ }^{\circ}\right)$, dimethyl fumarate $\left(\right.$ Tecfider $\left.{ }^{\circ}\right)$, fingolimod (Gilenya $\left.{ }^{\circ}\right)$, natalizumab (Tysabri $\left.{ }^{\circ}\right)$ and teriflunomide (Aubagio ${ }^{\circ}$ ). According to routine practice and recommendations by the German Society of Neurology [10] patient visits on which data will be collected are expected every 6 months.

The study was registered on 05 March 2018 in the EU PAS Register (http://www.encepp.eu/encepp/studiesDatabase.jsp) under the EU PAS Register Number EUPAS22951. Data will be collected at a planned 250 centers across Germany. The recruitment period will take 30 months. The observation period per patient will continue for 7.5 to 10 years, depending on the date of inclusion. The study started in April 2018 (first record of data on the first study subject in the study database). The end of study (last record of data on last study subject) is estimated for the third quarter of 2028 (Fig. 1). Visits follow routine practice and are planned to be documented approximately every 6 months for up to 10 years. Follow-up visits are planned for the entire study period for all participants, regardless of discontinuation of the studied medication or development of a malignancy.

CONFIDENCE will comply with Good Pharmacoepidemiological Practice [11] guidelines by the International Society of Pharmacoepidemiology (ISPE) and with national and EU requirements for ensuring the well-being and rights of participants in non-interventional PASSs. Ethics votes were obtained in accordance with local legal requirements for non-interventional studies in Germany (professional code of conduct of the medical associations). The independent ethics committee at the Technical University Desden has given the first professional advice for this observational study (Ethikkommission an der Technischen Universität Dresden, Germany; 12 February 2018 and 10 April 2019; reference EK 62022018). Obtaining further ethics votes was the individual responsibility of the participating physicians.

\section{Study population}

Data will be collected from 3000 patients with RMS or PPMS newly treated with ocrelizumab (ocrelizumab cohort; Fig. 1) and 1500 patients newly treated with selected MS DMTs other than ocrelizumab. Recruitment for both cohorts will take place at the same centers. To avoid over-representation of individual DMTs, a maximum of 500 patients will be included for each DMT other than ocrelizumab. This will be achieved by regularly monitoring the recruitment status and stopping enrolment of patients who receive the respective DMT when the maximum is reached.

Data from CONFIDENCE will be used in global postmarketing safety commitments, which, among other objectives, aim to investigate the occurrence of malignancies, particularly breast cancer, during ocrelizumab therapy. In order to adequately power breast cancer event rate analyses, at least $70 \%$ of each cohort will be female. This will be ensured by monitoring the recruitment status and stopping enrollment of male patients when their quota is reached.

Inclusion criteria include signed informed consent, diagnosis of MS, age $\geq 18$ years, and patients must be newly treated (for the first time during the course of their MS therapy) with ocrelizumab (for the ocrelizumab cohort) or the specific other DMTs (for the other DMT cohort) (up to 30 days prior to or 60 days after study enrollment) according to the local label irrespective of the reason for starting the treatment. Exclusion criteria include participation in an interventional study investigating MS DMTs, patients who have received ocrelizumab or their new MS DMT other than ocrelizumab more than 30 days prior to study entry, and patients who have been previously treated with rituximab. Patients who are 


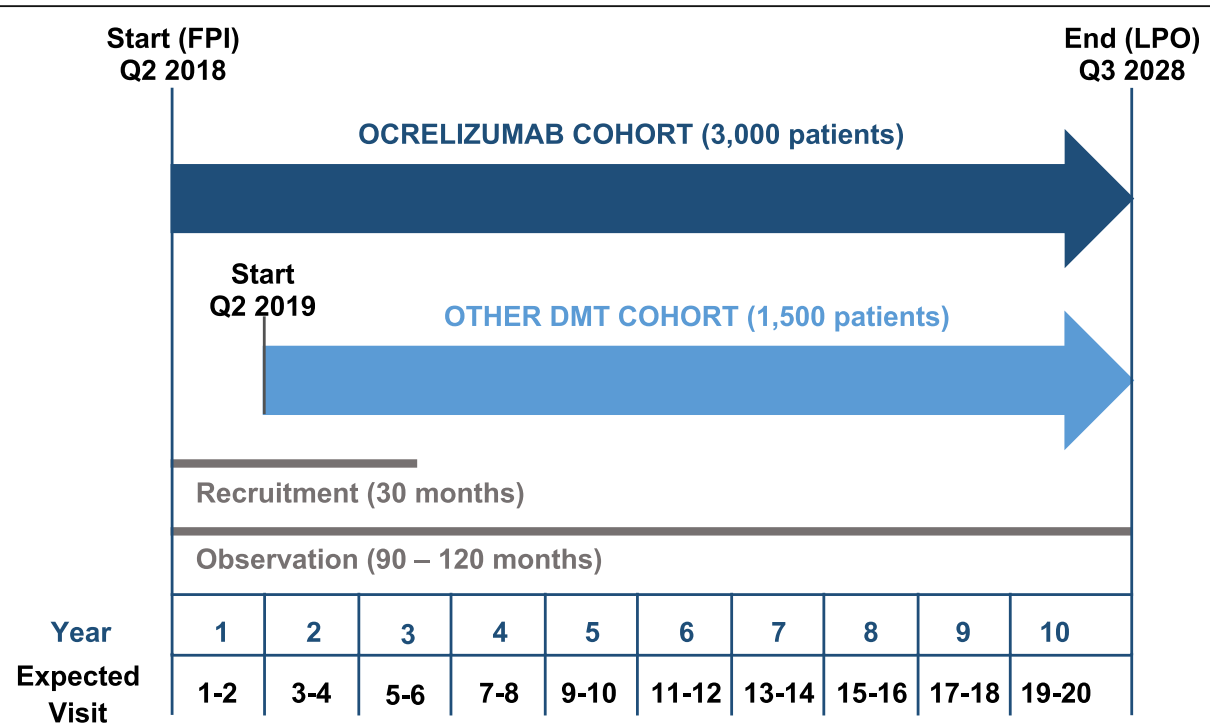

Fig. 1 Study design of CONFIDENCE. CONFIDENCE comprises two cohorts, the ocrelizumab cohort and the other DMT cohort. Recruitment into the ocrelizumab cohort started in April 2018, while recruitment into the other DMT cohort will start in Q2 2019. The observation period is planned to continue until the third quarter of 2028. Observation per patient will be 7.5 years ( 90 months) to 10 years ( 120 months; in the ocrelizumab cohort) depending on the time of inclusion. Visits follow routine practice and are expected to be documented every 6 months. FPI, first patient in; LPO, last patient out; Q, quarter

included in the 'other DMT cohort' must not have previously received ocrelizumab therapy.

\section{Endpoints}

All CONFIDENCE endpoints are listed in Table 1.

\section{Data collection}

Data collection according to standard of care starts after written informed consent by the patient and can take place before the first administration of ocrelizumab or other DMT (up to 30 days before or 60 days after study enrollment). Only available data will be collected; no additional diagnostic or monitoring procedures shall be applied to the patients outside of routine clinical practice. Data will be entered online by study site staff into electronic case report forms (eCRFs) based on the MS management system 3D (MSDS3D; MedicalSyn, Stuttgart, Germany) adapted for CONFIDENCE. This software had previously been developed to collect real-world safety data [12-14] and is well established as an eCRF tool in MS-related observational studies [15-18].

Data will be collected at screening, baseline, follow-up visits, and upon study completion/withdrawal as summarized in Table 2. As this is a non-interventional study, data are only documented if they are generated according to clinical routine; no examinations are carried out specifically for this study. There are no mandated study visits, and data from any encounter with the neurologist during follow-up will be entered (anticipated to occur every $\sim 6$ months). Follow-up is planned regardless of whether patients discontinue their current MS treatment.

Data sources include patient charts, interviews, medical examinations and questionnaires on patient reported outcomes (PROs; see below). The completion of such questionnaires is not routine practice but is allowed according to local regulations (published by a joint notification of the two German higher federal authorities [19];). Available clinical information on new malignancies, defined as the first occurrence of a cancer diagnosis during study followup including recurrences, will be provided by the treating physicians (treating neurologist and oncologist/pathologist). In addition data linkage with national and regional cancer registries will be explored to ensure identification of all malignancy cases in the study population.

This study uses five patient questionnaires to obtain data on PROs and on pharmacoeconomic outcomes: the Treatment Satisfaction Questionnaire for Medication (TSQM) 1.4 [20], the Multiple Sclerosis Impact Scale (MSIS)-29v2 [21], an adapted version (with a six-point improvement scale) of the Clinical Global Impression (CGI) [22], the Symbol Digit Modality Test (SDMT) [23], and the Work Productivity and Activity Impairment Questionnaire: Multiple Sclerosis (WPAI:MS) [24].

\section{Statistical analysis}

The sample size is planned to allow for the detection of AEs with an incidence of at least $0.1 \%$ ( 1 out of 1000 patients) at least once with a probability of $95 \%$. Applying the Poisson distribution model for uncommon events, 
Table 1 Safety, effectiveness and exploratory endpoints of CONFIDENCE

\begin{tabular}{|c|c|}
\hline $\begin{array}{l}\text { Primary safety endpoint } \\
\text { (ocrelizumab cohort) }\end{array}$ & $\begin{array}{l}\text { Incidence and type of uncommon AEs (including AEs with an incidence of } 0.1 \text { to } 1 \% \text { [ } 1 \text { to } 10 \text { out of } 1000 \\
\text { patients] or less) and death (including primary and underlying causes) in patients with MS newly exposed } \\
\text { to ocrelizumab }\end{array}$ \\
\hline $\begin{array}{l}\text { Key secondary safety endpoint } \\
\text { (other DMT cohort) }\end{array}$ & $\begin{array}{l}\text { Incidence and type of uncommon AEs (including AEs with an incidence of } 0.1 \text { to } 1 \% \text { [ } 1 \text { to } 10 \text { out of } 1000 \\
\text { patients] or less) and death (including primary and underlying causes) in patients with MS newly exposed } \\
\text { to selected DMTs other than ocrelizumab }\end{array}$ \\
\hline \multirow[t]{9}{*}{$\begin{array}{l}\text { Secondary effectiveness endpoints } \\
\text { (both cohorts) }\end{array}$} & $\begin{array}{l}\text { Treatment success: proportion of patients with no clinical disease activity measured by relapse and disease } \\
\text { progression and no discontinuation of current treatment due to AEs (excluding pregnancies) and lack of } \\
\text { effectiveness }\end{array}$ \\
\hline & Annualized relapse rate since start of treatment \\
\hline & Proportion of patients with relapses since start of treatment \\
\hline & Mean number of relapses per patients within the last 3 years before start of treatment \\
\hline & Change in expanded disability status scale (EDSS) from baseline \\
\hline & Proportion of patients with confirmed disability progression (CDP) \\
\hline & Time to onset of CDP \\
\hline & Time to onset of confirmed disability improvement (CDI) \\
\hline & Patient reported outcomes: treatment satisfaction, cognitive function, and health related quality of life \\
\hline \multirow{4}{*}{$\begin{array}{l}\text { Other safety endpoints (both } \\
\text { cohorts) }\end{array}$} & Incidence of all AEs \\
\hline & Incidence of serious infections \\
\hline & Incidence of malignancies \\
\hline & Incidence of mortality due to malignancies \\
\hline \multirow[t]{4}{*}{$\begin{array}{l}\text { Exploratory endpoints (both } \\
\text { cohorts) }\end{array}$} & $\begin{array}{l}\text { Mean number of gadolinium (Gd) enhancing } \mathrm{T} 1 \text { lesions as detected by brain magnetic resonance imaging } \\
\text { (MRI) }\end{array}$ \\
\hline & Change from baseline in MRI lesions (T2 and Gd + lesions) \\
\hline & $\begin{array}{l}\text { Global impression on the disease course as reported by physician and patient using an adapted 'Clinical } \\
\text { Global Impression (CGI)' scale: Severity Score (change from baseline) and Improvement Score (by descriptive } \\
\text { statistics [mean, standard deviation]) }\end{array}$ \\
\hline & $\begin{array}{l}\text { Change from baseline in pharmacoeconomic outcomes as measured by 'Work Productivity and Activity } \\
\text { Impairment Questionnaire: Multiple Sclerosis (WPAl:MS)' }\end{array}$ \\
\hline
\end{tabular}

the total sample size results in approximately 3000 patients - the size of the ocrelizumab cohort. Taking into account that ocrelizumab is currently the only approved disease modifying medication for PPMS and the resulting unmet need in this population, approximately 7001000 patients in the ocrelizumab cohort are expected to be PPMS patients. The other DMT cohort of 1500 patients will allow for the detection of AEs with an incidence rate of at least $0.2 \%$ ( 1 out of 500 patients) at least once with a probability of $95 \%$.

Statistical analyses will be mainly descriptive and exploratory in nature. Statistical modeling and inferential statistics will also be used, but will be interpreted in an exploratory fashion. Missing data will not be substituted ('observed cases analysis'). Three analysis sets will be used: the Enrolled Set (ES; all patients recorded in the clinical database who have given informed consent), the Safety Set (SS; all patients in the ES treated with at least one dose of the studied medicinal product documented in the eCRF), and the Full Analysis Set (FAS; all patients of the SS who had at least one documentation after start of therapy). Based on patients'potential switch of treatment, four subsets will be derived from the SS and FAS set separately. These are: patients who receive ocrelizumab during the whole course of the study plus patients who switch from ocrelizumab to DMT until this treatment switch (subset 1), patients who receive DMT during the whole course of the study plus patients who switch from DMT to ocrelizumab until this treatment switch (subset 2), patients who switch from DMT to ocrelizumab, after treatment switch (subset 3), and patients who switch from ocrelizumab to DMT, after treatment switch (subset 4).

All effectiveness analyses will be performed separately for each combination of cohort (ocrelizumab and other MS DMTs), MS type (RMS and PPMS) and FAS subset 1 to 4 . Similarly, all safety analyses will be performed separately for each combination of cohort (ocrelizumab and other MS DMTs), MS type (RMS, PPMS, and total) and SS subset 1 to 4 . Subgroup analyses will comprise EDSS at baseline (0-3.5 / $\geq 4$ for RMS, 0-5.5 / > 5.5-6.5 $/ \geq 7$ for PPMS), MS-specific pre-treatment, age (<40 / $\geq 40$ years for RMS, $\leq 40$ / 41-55 / > 55 years for PPMS), sex (male vs. female). 
Table 2 Data collection overview

\begin{tabular}{|c|c|c|c|c|}
\hline Data to be collected & $\begin{array}{l}\text { Study } \\
\text { Enrollment } \\
\text { (Screening) }\end{array}$ & $\begin{array}{l}\text { Baseline } \\
\text { (Study } \\
\text { Entry) }\end{array}$ & $\begin{array}{l}\text { Data Collection } \\
\text { (approximately every } \\
6 \text { months) }\end{array}$ & $\begin{array}{l}\text { Study Completion/ } \\
\text { Study Withdrawal }\end{array}$ \\
\hline Patient demographics & $x$ & & & \\
\hline Informed consent & $x$ & & & \\
\hline Inclusion/exclusion criteria & $x$ & & & \\
\hline Vital signs and measurements ${ }^{a}$ & & $x$ & $x$ & $x$ \\
\hline Pregnancy status (patient-reported) & & $x$ & $x$ & $x$ \\
\hline Pregnancies & & wheneve & hey occur & \\
\hline Laboratory test results $^{\mathrm{b}}$ & & $x$ & $x$ & $x$ \\
\hline John Cunningham Virus (JCV) antibody status / index & & $x$ & & \\
\hline MS disease history ${ }^{c}$ & & $x$ & & \\
\hline MS treatment history ${ }^{d}$ & & $x$ & & \\
\hline Medical history and comorbidities & & $x$ & & \\
\hline $\begin{array}{l}\text { Clinically significant medical and surgical history (including previous } \\
\text { malignancies and precancerous lesions) }\end{array}$ & & $x$ & & \\
\hline Malignancy risk factor information ${ }^{\mathrm{e}}$ & & $x$ & & \\
\hline Cancer screening $^{f}$ & & $x$ & $x$ & $x$ \\
\hline MS symptoms ${ }^{9}$ & & $x$ & $x$ & $x$ \\
\hline Current MS DMT administration information ${ }^{\text {h }}$ & & $x$ & $x$ & $x$ \\
\hline $\begin{array}{l}\text { Patient Reported Outcomes (PROs): Treatment Satisfaction Questionnaire } \\
\text { for Medication (TSQM) 1.4, Multiple Sclerosis Impact Scale (MSIS)-29v2, } \\
\text { adapted Clinical Global Impression (CGI), Symbol Digit Modality Test } \\
\text { (SDMT) }\end{array}$ & & $x$ & $x$ & $x$ \\
\hline $\begin{array}{l}\text { Pharmacoeconomic outcomes: Work Productivity and Activity } \\
\text { Impairment Questionnaire: MS (WPAl:MS) }\end{array}$ & & $x$ & $x$ & $x$ \\
\hline Premature termination incl. Reasons for study withdrawal & & & & $x$ \\
\hline Continuation of therapy or reasons for treatment discontinuation & & & $x$ & $x$ \\
\hline $\begin{array}{l}\text { Prior and concomitant medications other than MS medication (up to } 3 \\
\text { months prior to study entry) }\end{array}$ & & $x$ & $x$ & $x$ \\
\hline Any malignancies ${ }^{i}$ & & wheneve & hey occur & \\
\hline $\begin{array}{l}\text { All serious and non-serious AEs incl. Non-melanoma skin cancer (NMSCs) } \\
\text { and information on reasons for death }\end{array}$ & & wheneve & hey occur & \\
\hline Death information & & & $x$ & $x$ \\
\hline
\end{tabular}

${ }^{a}$ Height, weight, blood pressure, heart rate and temperature

b Blood cell count, immunoglobulins, liver enzymes, renal status, clinical chemistry, flow cytometry. Viral serology (only at baseline)

c Including MS date of diagnosis, type of MS, duration of MS, MS disease symptom history, relapse history, EDSS (or proxy) and MRI results (if available)

${ }^{d}$ Prior use and duration of therapies for MS and prior use and duration of immunomodulatory, immunosuppressive, and anti-neoplastic agents (if any)

e Including tobacco use history, alcohol use history, family disease history, genetic testing such as BRCA1 and 2, personal history of malignancy, or other risk factors including reproductive history (women and undifferentiated only); the selection of these risk factors is based on the VERISMO study (for further details on VERISMO, see Discussion)

${ }^{f}$ Including gynecological consultation, breast check, dermatological check, or other malignancy/cancer screening tests and procedures (e.g., mammography, Pap test, colonoscopy, laboratory malignancy markers)

${ }^{\mathrm{g}}$ Including MS relapse during treatment period, MS type changes, date of last administration of Ocrelizumab (if applicable), MS DMT changes and rationale, most recent EDSS score since last encounter (if available), most recent MRI results since last encounter (if available)

${ }^{\mathrm{h}}$ Dates of administration (start, stop, and restart dates), dose, dosing frequency, reason for discontinuation (if applicable)

' Medical records regarding in-depth malignancy information will be solicited from the patient's oncologist or pathologist (only collected if a malignancy

is identified)

For continuous data, the mean, standard deviation, median, range (minimum, maximum), and interquartile range (Q1, Q3) will be calculated. Categorical data will be displayed by absolute and relative frequencies. Incident rates will be calculated for safety endpoints. Timeto-event endpoints will be analyzed using Kaplan-Meier product limit methods to estimate the survival distribution curves and the median survival time. A negative binomial model will investigate the annualized relapse rates. The number/percentage of patients with a change in MS / RMS type at any time during the study will be summarized and the time to change in years will be 
calculated. The number/percentage of patients with relapses any time and per visit during study will be calculated. Methods of survival analysis (Kaplan-Meier summary and corresponding Kaplan-Meier plot) will be performed. These also will be repeated for all subgroups. A logistic regression model will be used to evaluate in how far the probability for treatment success is influenced by covariates like EDSS baseline score, age (continuous), sex, categories based on enrolment date, MS type. The full model is specified in the statistical analysis plan. Treatment exposure, the number of administrations per patient and the total dose per patient will be summarized as numeric variables. The analysis on treatment exposure will be repeated for all subgroups. Annual interim analyses are planned starting when 1-year data of at least 500 patients are available.

\section{Discussion}

\section{Strengths of this study}

Data collected from the pivotal randomized clinical trials (RCTs) provide a sound body of evidence for the efficacy and safety of ocrelizumab treatment for RMS and PPMS $[6,7]$ and led to the approval of ocrelizumab. CONFIDENCE is a large, non-interventional study with less restrictive enrolment criteria, greater patient number and longer observation periods than the pivotal studies. The real-world evidence obtained in CONFIDENCE will help answer questions that cannot be answered by RCTs, question relevant for daily practice, the medical community and health insurance [25].

CONFIDENCE offers the possibility to detect rare safety events in enrolled patients regardless of a change in treatment or discontinuation of ocrelizumab. The comprehensive collection of risk factors and an adjudication process for newly occurred malignancies facilitates the reliable interpretation of malignancy events. This will be a solid basis for the investigation of a possible effect of treatment on the occurrence of cancer. Furthermore, this study will help learn more about the treatment algorithm of MS patients, because it collects data on underlying reasons for therapy changes and discontinuations.

\section{Minimization of limitations}

Rather than observing ocrelizumab in isolation, the study aims to present ocrelizumab data in the context of the safety profiles of other selected approved MS DMTs. Consequently, a cohort of patients newly treated during the course of their MS therapy with alemtuzumab, cladribine, dimethyl fumarate, fingolimod, natalizumab, or teriflunomide will be observed in parallel with the ocrelizumab cohort. However, label differences between ocrelizumab and other DMTs are potential confounding factors. In order to be able to minimize such potential confounding factors and to take different prerequisites

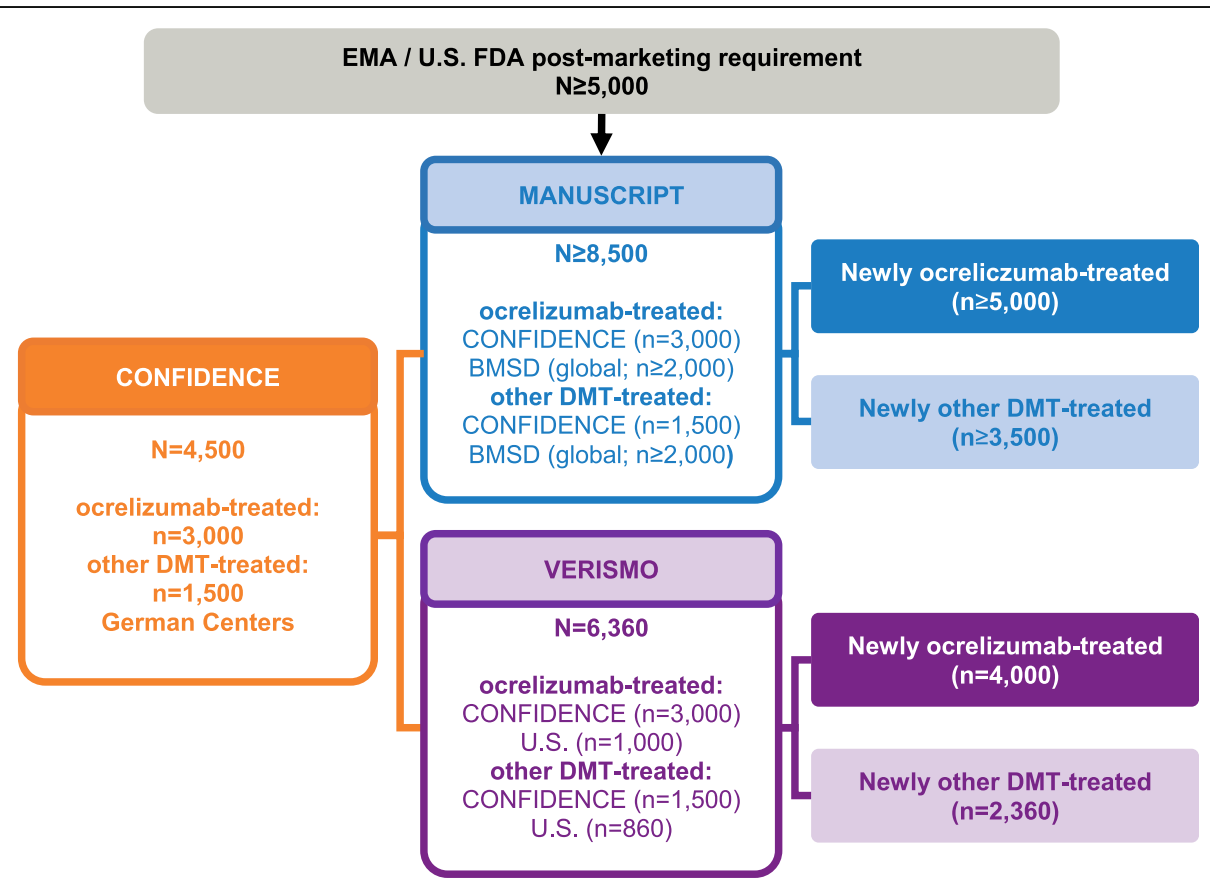

Fig. 2 Integration of safety data from CONFIDENCE into other global post-marketing safety studies. Safety data from the German study CONFIDENCE will be integrated into the global post-marketing safety studies MANUSCRIPT and VERISMO. MANUSCRIPT (blue) will combine data from CONFIDENCE (orange) and from multiple existing international MS and population registries (Big MS Data Group, BMSD, which combines clinical data from multiple registries, i.e. MSBase, France OFSEP [Observatoire Français de la Sclérose en Plaques], Denmark, Sweden and Italy). VERISMO (purple) will combine prospective data collected at sites in the United States (U.S.) and the German CONFIDENCE sites 
for individual drug therapies into account, factors associated with treatment choice (e.g. history of relapse and MRI activity as well as the most recent EDSS) will be collected at study enrollment.

To address potential enrollment bias between cohorts, sites are encouraged to enroll patients into both treatment cohorts and to include patients representing the site's general population regarding patient disposition and treatment regimen. Patients will be enrolled as new users at the time of ocrelizumab or other MS DMT treatment initiation; this will reduce potential bias (healthy user bias, depletion of susceptibles) associated with the study of long-term medication users. This will also ensure that the same starting point applies to each drug treatment and that all side effects can be recorded including those that arise from at least one dose. To reduce possible misclassification, all sites will undergo standardized training and utilize standardized documentation for the completion of eCRFs at enrollment and for each follow-up assessment, specifically on collecting exposure and outcome variable information.

The willingness to introduce new drugs such as ocrelizumab into MS therapy is difficult to predict and may affect the achievement of recruitment targets. Continuous monitoring of patient enrollment at the site and country level will allow a rapid response to underrecruiting, e.g. by initiation of additional sites.

\section{CONFIDENCE as a central element of the global ocrelizumab post-marketing safety program}

In order to fulfil regulatory requirements of the FDA and the EMA, a program comprising two observational studies - VERISMO (EUPAS30752) and MANUSCRIPT (EUPAS28619) - has been developed to characterize the long-term safety of ocrelizumab in the post-marketing setting. VERISMO and MANUSCRIPT are multi-source, international, non-interventional, longitudinal cohort post-marketing safety studies involving patients with MS who have newly initiated treatment with ocrelizumab and other MS DMTs [26]. VERISMO aims to determine the incidence rate of breast cancer and all malignancies, is expected to enroll a total of 6360 patients (including 4000 patients treated with ocrelizumab; Fig. 2), and collects prospective data at study sites in the US and Germany. MANUSCRIPT aims to estimate rates of serious AEs, including malignancies and serious infection. It is expected to collect data from at least $8500 \mathrm{MS}$ patients (including $\geq 5000$ patients treated with ocrelizumab; Fig. 2), and is primarily based on data from multiple existing international MS and population registries (i.e. Big MS Data Group, BMSD).

CONFIDENCE is aligned with the FDA and EMA commitments, and safety data collected in CONFIDENCE will be integrated in VERISMO and MANUSCRIPT.
Therefore, CONFIDENCE is the central study in the global ocrelizumab post-marketing safety program (Fig. 2). All pregnancies observed within CONFIDENCE will be additionally transferred to the German nationwide Multiple Sclerosis and Pregnancy Registry (Ruhr-University Bochum) and the Ocrevus Pregnancy Registry (EU PAS register number not available yet) if the pregnant woman has given her informed consent. In contrast to VERISMO and MANUSCRIPT, CONFIDENCE also investigates a number of effectiveness endpoints (Table 1), which distinguishes it from the other two studies.

\section{Abbreviations \\ AE: Adverse event; BMSD: Big MS Data Group; CDI: Confirmed disability improvement; CDP: Confirmed disability progression; CGI: Clinical Global Impression; CNS: Central nervous system; DMT: Disease modifying therapy; eCRF: Electronic Case report form; EDSS: Expanded disability status scale; EMA: European Medicines Agency; FDA: Food and Drug Administration; FPI: First patient in; Gd: Gadolinium; GPP: Good Pharmacoepidemiological Practice; IFN: Interferon; IgG1: Immnunoglobulin G1; ISPE: International Society of Pharmacoepidemiology; JCV: John Cunningham Virus; LPO: Last patient out; MRI: Magnetic resonance imaging; MS: Multiple sclerosis; MSDS3D: Multiple sclerosis management system 3D; MSIS: Multiple Sclerosis Impact Scale; NMSC: Non-melanoma skin cancer; OFSEP: Observatoire Français de la Sclérose en Plaques; PASS: Post-authorization safety study; PPMS: Primary progressive multiple sclerosis; PRO: Patient reported outcome; RMS: Relapsing multiple sclerosis; RRMS: Relapsing-remitting Multiple Sclerosis; SDMT: Symbol Digit Modality Test; SmPC: Summary of Product Characteristics; TSQM: Treatment Satisfaction Questionnaire for Medication; U.S.: United States; WPAl:MS: Work Productivity and Activity Impairment Questionnaire: Multiple Sclerosis}

\section{Acknowledgements}

The authors thank the participating physicians and centers and the current and future patients participating in this study. The CONFIDENCE study is sponsored by Roche Pharma AG (Grenzach-Wyhlen, Germany). Medical writing support for this manuscript was provided by AMS Advanced Medical Services GmbH (Mannheim, Germany) and Physicians World Europe GmbH (Mannheim, Germany).

\section{Authors' contributions}

$P D, V Z, J L, H B, S H S, D W, T Z$ : All were involved in the conception and design of the study and drafting and revision of the manuscript. PD, VZ, JL, HB, SHS, DW, TZ: All approved the final version of the manuscript.

\section{Funding}

This work was financially supported by Roche Pharma AG, Grenzach-Wyhlen, Germany. The funding body participated in the design of the study and sponsored writing of the manuscript. The authors were responsible for the decision to publish as well as for review and revision of the manuscript.

\section{Availability of data and materials}

Qualified researchers may request access to individual patient level data through the clinical study data request platform (www.

clinicalstudydatarequest.com). Further details on Roche's criteria for eligible studies are available here (https://clinicalstudydatarequest.com/StudySponsors/Study-Sponsors-Roche.aspx). For further details on Roche's Global Policy on the Sharing of Clinical Information and how to request access to related clinical study documents, see here (https://www.roche.com/research_ and_development/who_we_are_how_we_work/clinical_trials/our_ commitment_to_data_sharing.htm).

\section{Ethics approval and consent to participate}

CONFIDENCE was evaluated by the Ethics Committee at the Technical University of Dresden, Germany (Ethikkommission an der Technischen Universität Dresden, Germany; 12 February 2018 and 10 April 2019; reference EK 62022018). All patients have to give signed informed consent to participate in the study. 


\section{Consent for publication}

Not applicable.

\section{Competing interests}

PD is an employee of Roche Pharma AG. VZ is an employee of F. HoffmannLa Roche AG. JL and HB are employees of Roche Pharma AG and shareholders of F. Hoffmann-La Roche AG. SHS is employee of Roche Pharma AG. DW is an employee and shareholder of F. Hoffmann-La Roche AG. TZ has received grants and personal fees from Biogen, Novartis, Sanofi and Teva, and personal fees from Almirall, Bayer, F. Hoffmann-La Roche AG and Merck; he is a member of the editorial board (Associate Editor) of BMC Neurology. All authors received support for their contribution to this study as well as medical writing support for the development of this manuscript from Roche Pharma AG, Grenzach-Wyhlen, Germany.

\section{Author details}

'Roche Pharma AG, Emil-Barell-Straße 1, 79639 Grenzach-Wyhlen, Germany. 2F. Hoffmann-La Roche Ltd, Grenzacherstraße 124, 4070 Basel, Switzerland.

${ }^{3}$ Universitätsklinikum Carl Gustav Carus, Zentrum für klinische

Neurowissenschaften, Fetscherstr. 74, 01307 Dresden, Germany.

\section{Received: 25 July 2019 Accepted: 28 February 2020}

\section{1. .}

\section{References}

1. Tullman MJ. Overview of the epidemiology, diagnosis, and disease progression associated with multiple sclerosis. Am J Manag Care. 2013;19(2 Suppl):S15-20

2. Du Pasquier RA, Pinschewer DD, Merkler D. Immunological mechanism of action and clinical profile of disease-modifying treatments in multiple sclerosis. CNS Drugs. 2014;28(6):535-58. https://doi.org/10.1007/s40263-014-0160-8.

3. Rae-Grant A, Day GS, Marrie RA, Rabinstein A, Cree BAC, Gronseth GS, et al. Practice guideline recommendations summary: disease-modifying therapies for adults with multiple sclerosis: report of the guideline development, dissemination, and implementation subcommittee of the American Academy of Neurology. Neurology. 2018;90(17):777-88. https://doi.org/10. 1212/WNL.0000000000005347.

4. Rommer PS, Zettl UK. Managing the side effects of multiple sclerosis therapy: pharmacotherapy options for patients. Expert Opin Pharmacother. 2018;19(5):483-98. https://doi.org/10.1080/14656566.2018.1446944.

5. Klein C, Lammens A, Schäfer W, Georges G, Schwaiger M, Mössner E, et al. Epitope interactions of monoclonal antibodies targeting CD20 and their relationship to functional properties. mAbs. 2013;5(1):22-33. https://doi.org/ 10.4161/mabs.22771

6. Hauser SL, Bar-Or A, Comi G, Giovannoni G, Hartung HP, Hemmer B, et al. Ocrelizumab versus interferon beta-1a in relapsing multiple sclerosis. N Engl J Med. 2017;376(3):221-34. https://doi.org/10.1056/NEJMoa1601277.

7. Montalban X, Hauser SL, Kappos L, Arnold DL, Bar-Or A, Comi G, et al. Ocrelizumab versus placebo in primary progressive multiple sclerosis. $\mathrm{N}$ Engl J Med. 2017:376(3):209-20. https://doi.org/10.1056/NEJMoa1606468.

8. Kappos L, Li D, Calabresi PA, O'Connor P, Bar-Or A, Barkhof F, et al. Ocrelizumab in relapsing-remitting multiple sclerosis: a phase 2, randomised, placebo-controlled, multicentre trial. Lancet. 2011;378(9805): 1779-87. https://doi.org/10.1016/S0140-6736(11)61649-8.

9. Hauser L, Kappos L, Montalban X, Hughes R, Koendgen H, McNamara J, et al. Safety of ocrelizumab in multiple sclerosis: Updated analysis in patients with relapsing and primary progressive multiple sclerosis. ECTREMIS Online Library 2018, 229069. https://onlinelibrary.ectrims-congress.eu/ ectrims/2018/ectrims-2018/229069/stephen.I.hauser.safety.of.ocrelizumab.in multiple.sclerosis.updated.analysis.html. Accessed 15 Apr 2019.

10. DGN Leitlinie zur Diagnose und Therapie der MS (Guidelines for MS diagnosis and treatment by the German Society of Neurology). https:// www.dgn.org/leitlinien/2333-II-31-2012-diagnose-und-therapie-dermultiplen-sklerose. 21.08.2015. Accessed 08 Feb 2019

11. Guidelines for Good Pharmacoepidemiology Practices (GPP), 2015. https:// www.pharmacoepi.org/resources/policies/guidelines-08027/. Accessed 08 Feb 2019

12. Haase R, Wunderlich M, Dillenseger A, Kern R, Akgün K, Ziemssen T. Improving multiple sclerosis management and collecting safety information in the real world: the MSDS3D software approach. Expert Opin on Drug Saf. 2018;17(4):369-78. https://doi.org/10.1080/14740338.2018.
13. Kern R, Haase R, Eisele JC, Thomas K, Ziemssen T. Designing an electronic patient management system for multiple sclerosis: building a next generation multiple sclerosis documentation system. Interact J Med Res. 2016:5(1):e2. https://doi.org/10.2196/ijmr.4549.

14. Ziemssen T, Kempcke R, Eulitz M, Großmann L, Suhrbier A, Thomas K, et al. Multiple sclerosis documentation system (MSDS): moving from documentation to management of MS patients. J Neural Transm. 2013; 120(Suppl 1):S61-6. https://doi.org/10.1007/s00702-013-1041-X.

15. Ziemssen $T$, Kern $R$, Cornelissen C. The PANGAEA study design - a prospective, multicenter, non-interventional, long-term study on fingolimod for the treatment of multiple sclerosis in daily practice. BMC Neurol. 2015; 15:93. https://doi.org/10.1186/s12883-015-0342-0.

16. Ziemssen T, Engelmann U, Jahn S, Leptich A, Kern R, Hassoun L, et al. Rationale, design, and methods of a non-interventional study to establish safety, effectiveness, quality of life, cognition, health-related and work capacity data on Alemtuzumab in multiple sclerosis patients in Germany (TREAT-MS). BMC Neurol. 2016;16:109. https:/doi.org/10.1186/s12883-016-0629-9.

17. Ziemssen T, Gass A, Wuerfel J, Bayas A, Tackenberg B, Limmroth V, et al. Design of TRUST, a non-interventional, multicenter, 3-year prospective study investigating an integrated patient management approach in patients with relapsing-remitting multiple sclerosis treated with natalizumab. BMC Neurol. 2016;16:98. https://doi.org/10.1186/s12883-016-0625-0.

18. Ziemssen T, Kern R, Cornelissen C. Study design of PANGAEA 2.0, a noninterventional study on RRMS patients to be switched to fingolimod. BMC Neurol. 2016;16:129. https://doi.org/10.1186/s12883-016-0648-6.

19. BfArM and PEl. Empfehlungen des Bundesinstituts für Arzneimittel und Medizinprodukte und des Paul-Ehrlich-Instituts zur Planung, Durchführung und Auswertung von Anwendungsbeobachtungen (AWB) vom 7. Juli 2010. https://www.pei.de/SharedDocs/Downloads/pu/klinische-pruefung/100707awb-kommentierung-fachkreise.pdf?_blob=publicationFile\&v=1. German. Accessed 13 Mar 2019

20. Atkinson MJ, Sinha A, Hass SL, Colman SS, Kumar RN, Brod M, et al. Validation of a general measure of treatment satisfaction, the treatment satisfaction questionnaire for medication (TSQM), using a national panel study of chronic disease. Health Qual Life Outcomes. 2004;2:12.

21. Hobart J, Lamping D, Fitzpatrick R, Riazi A, Thompson A. The multiple sclerosis impact scale (MSIS-29). A new patient-based outcome measure. Brain. 2001;124:962-73.

22. Guy W. Editor. ECDEU assessment manual for psychopharmacology. Rockville: US Department of Heath, Education, and Welfare Public Health Service Alcohol, Drug Abuse, and Mental Health Administration; 1976.

23. Benedict RHB, DeLuca J, Phillips G, LaRocca N, Hudson LD, Rudick R, Multiple sclerosis outcome assessments consortium. Validity of the symbol digit modalities test as a cognition performance outcome measure for multiple sclerosis. Mult Scler. 2017;23:721-33. https://doi.org/10.1177/ 1352458517690821.

24. Reilly MC, Zbrozek AS, Dukes EM. The validity and reproducibility of a work productivity and activity impairment instrument. PharmacoEconomics. 1993; 4(5):353-65

25. Tjalf Ziemssen, Jan Hillert, Helmut Butzkueven. The importance of collecting structured clinical information on multiple sclerosis. BMC Med. 2016;14(1).

26. Ziemssen T, Berthold H, Dirks P, Evershed J, Gunzenhauser K, Leemhuis J, et al. Integration of ocrelizumab safety data from the German study CONFIDENCE into the global post-marketing safety studies MANUSCRIPT and VERISMO. ECTRIMS Online Library 2018, 229464. https://onlinelibrary. ectrims-congress.eu/ectrims/2018/ectrims-2018/229464/tjalf.ziemssen. integration.of.ocrelizumab.safety.data.from.the.german.study.html. Accessed 15 Apr 2019.

\section{Publisher's Note}

Springer Nature remains neutral with regard to jurisdictional claims in published maps and institutional affiliations. 